\title{
Contrast enhanced magnetic resonance imaging of the terminal ileum in children with Crohn's disease
}

\author{
A Laghi, O Borrelli, P Paolantonio, L Dito, M Bueno de Mesquita, P Falconieri, \\ R Passariello, S Cucchiara
}

Gut 2003;52:393-397

See end of article for authors' affiliations

Correspondence to: Dr S Cucchiara Department of Paediatric Gastroenterology, Head University of Rome "La Sapienza", Viale Regina Elena 324, 00161 Rome, Italy;

salvatore.cucchiara@ uniromal it

Accepted for publication 20 September 2002
Background and aims: Recently, magnetic resonance imaging (MRI) has been introduced in the diagnosis of patients with inflammatory bowel disease (IBD). However, it is still rarely reported in paediatric IBD. We studied the diagnostic value of gadolinium enhanced MRI in revealing inflammation of the distal ileum in children with Crohn's disease (CD) and in differentiating them from patients with other inflammatory diseases of the gut. MRI was performed using a polyethylene glycol (PEG) solution as oral contrast agent to distend the small bowel (CE-PEG-MRI).

Subjects and methods: Seventy five consecutive patients (median age 13.6 years, range 8-17) with suspected CD underwent ileocolonoscopy with biopsy and CE-PEG-MRI. CD activity was measured by the paediatric Crohn's disease activity index (PCDAI). CE-PEG-MRI was evaluated with an overall score calculated, taking into account both wall thickness and contrast enhancement.

Results: Active CD with distal ileitis was diagnosed in 26 cases, active ulcerative colitis (UC) in 18 and spondyloarthropathy and indeterminate ileocolitis in 11;20 children served as controls. In all CD patients, CE-PEG-MRI revealed a marked ileal involvement with increased wall thickness and parietal contrast enhancement and showed a high concordance with endoscopy and histology, whereas the test was negative in all controls. Of the $18 \mathrm{UC}$ patients, CE-PEG-MRI was negative in 15 and showed a mild parietal contrast enhancement of the terminal ileum in only three of seven patients with backwash ileitis. Among the group of spondyloarthropathy patients, six had mucosal erosions and five mild superficial ileitis: CE-PEG-MRI was negative in four and revealed only mild parietal contrast enhancement of the ileal wall in seven. CE-PEG-MRI did not show an increase in wall thickness of the dista ileum in any of the UC or spondyloarthropathy patients. The sensitivity and specificity of CE-PEG-MRI related to the presence of erosive ileitis, as documented by endoscopy, were $84 \%$ and $100 \%$, respectively. In addition, the test correlated markedly with endoscopy and histology in the entire population $(r=0.94 ; r=0.95$, respectively) as well as with the PCDAl in CD patients $(r=0.91)$.

Conclusions: In children with active CD, CE-PEG-MRI is a very sensitive and specific test for the detection of distal ileitis and for differentiation from other inflammatory diseases of the gut. The test could also be useful for the firstline diagnostic approach in children with suspected CD. The high correlation of CE- PEG-MRI with ileal endoscopy and histology as well as with PCDAI makes this test of great interest for future studies as a tool for monitoring the clinical course and the effect of therapy in CD patients.
$\mathrm{R}$ ecent epidemiological data indicate an increased incidence of inflammatory bowel disease (IBD) in childhood (approximately $5.3 / 10^{5}$ children aged less than 16 years), with Crohn's disease (CD) being twice as common as ulcerative colitis (UC). ${ }^{12}$ While the two forms of IBD in children are characterised by overlapping symptoms and clinical signs, diagnosis and differentiation between them are based on the combination of endoscopy, histology, and radiology. ${ }^{3}$

New non-invasive methods such as leucocyte scintigraphy and sonography have been proposed as valuable tests in IBD patients both to detect bowel inflammation and to assess disease distribution and activity. ${ }^{45}$ Magnetic resonance imaging (MRI) with the use of an oral contrast agent has also been proposed as a useful test in adults with IBD, ${ }^{67}$ whereas its use is still rarely reported in children with IBD. ${ }^{8}$

The aim of this prospective study was to evaluate the diagnostic usefulness of gadolinium enhanced MRI in consecutive children with suspected CD in order to detect ileal inflammation and to differentiate CD from other forms of intestinal inflammation. Gadolinium enhanced MRI was performed using polyethylene glycol (PEG) solution as oral contrast agent for small bowel distension (CE-PEG-MRI) and com- pared with endoscopy and histology, the gold standard diagnostic procedures in IBD patients.

\section{MATERIALS AND METHODS}

The study was carried out in 75 consecutive children (median age 13.6 years, range 8-17) referred to the Paediatric Gastroenterology Unit, University of Rome, for suspected CD, from October 2001 to June 2002. Systemic disorders, infectious diseases, and food enteropathy were excluded in all. In each patient the entire colon and the last $20-25 \mathrm{~cm}$ of the terminal ileum were explored by means of a paediatric video colonoscope (Fujinon, Rome, Italy) after intravenous sedation with meperidine $(1-2 \mathrm{mg} / \mathrm{kg})$ and midazolam $(0.05 \mathrm{mg} / \mathrm{kg}$ up to a maximal $0.2 \mathrm{mg} / \mathrm{kg}$ or $5 \mathrm{mg}$ ). Well established endoscopic and histological criteria for diagnosis of $\mathrm{CD}$ were used. ${ }^{3}$ To analyse the data the following endoscopic score of the distal ileum was used: $0=$ no mucosal changes; $1=$ erythema,

Abbreviations: CD, Crohn's disease; UC, ulcerative colitis CE-PEG-MRI, contrast enhanced polyethylene glycol magnetic resonance imaging: $\mathrm{SpA}$, spondyloarthropathy; IBD, inflammatory bowel disease; PCDAl, paediatric Crohn's disease activity index. 
Table 1 Scoring system for histological assessment (modified from D'Haens and colleagues')

\begin{tabular}{|c|c|c|}
\hline Epithelial damage & $\begin{array}{l}0 \\
1 \\
2\end{array}$ & $\begin{array}{l}\text { Normal } \\
\text { Focal pathology } \\
\text { Extensive pathology }\end{array}$ \\
\hline Architectural changes & $\begin{array}{l}0 \\
1 \\
2\end{array}$ & $\begin{array}{l}\text { Normal } \\
\text { Moderately disturbed }(<50 \%) \\
\text { Severely disturbed }(>50 \%)\end{array}$ \\
\hline $\begin{array}{l}\text { Infiltration of mononuclear cells in } \\
\text { the lamina propria }\end{array}$ & $\begin{array}{l}0 \\
1 \\
2\end{array}$ & $\begin{array}{l}\text { Normal } \\
\text { Moderately increase } \\
\text { Severely increase }\end{array}$ \\
\hline $\begin{array}{l}\text { Infiltration of polymorphonuclear } \\
\text { cells in the lamina propria }\end{array}$ & $\begin{array}{l}1 \\
2 \\
3\end{array}$ & $\begin{array}{l}\text { Normal } \\
\text { Moderately increase } \\
\text { Severely increase }\end{array}$ \\
\hline $\begin{array}{l}\text { Polymorphonuclear cells in } \\
\text { epithelium }\end{array}$ & $\begin{array}{l}0 \\
1 \\
2\end{array}$ & $\begin{array}{l}\text { In surface epithelium } \\
\text { Cryptitis } \\
\text { Crypt abscess }\end{array}$ \\
\hline Presence of erosions and/or ulcers & $\begin{array}{l}0 \\
1\end{array}$ & $\begin{array}{l}\text { Yes } \\
\text { No }\end{array}$ \\
\hline Presence of granuloma & $\begin{array}{l}0 \\
1\end{array}$ & $\begin{array}{l}\text { Yes } \\
\text { No }\end{array}$ \\
\hline No of biopsy specimens affected & $\begin{array}{l}0 \\
1 \\
2 \\
3\end{array}$ & $\begin{array}{l}\text { None }(0 / 3) \\
\leqslant 33 \%(1 / 3) \\
33-66 \%(2 / 3) \\
>66 \%(3 / 3)\end{array}$ \\
\hline
\end{tabular}

oedema, and loss of vascular pattern; 2 =granularity and/or friability, and aphthoid erosions; $3=$ aphthoid or serpiginous ulcers, and cobblestoning; and $4=$ confluent serpiginous ulcers, strictures, and an abnormal lumen profile. A minimum of three biopsies were taken from the most inflamed areas of the terminal ileum. Biopsies were stained with haematoxylin and eosin and examined by an experienced gastrointestinal pathologist, unaware of the endoscopic features. Histological assessment of the terminal ileum was performed according to a score previously reported (table 1). ${ }^{9}$ Disease activity in patients with CD was measured using the paediatric Crohn's disease activity index (PCDAI). ${ }^{10}$

CE-PEG-MRI of the small bowel was performed in all patients at $1.5 \mathrm{~T}$ (Magnetom Vision Plus; Siemens Medical Systems, Erlangen, Germany) as previously described. ${ }^{11}$ Patients were studied in the supine position using a phased array body coil. After an overnight fast and immediately before the MRI examination, each patient was asked to drink continuously a PEG solution ( $10 \mathrm{ml} / \mathrm{kg}$ body weight). The latter was freshly prepared before administration by dissolving a granular powder containing $34.8 \mathrm{~g}$ PEG 4000, $1.42 \mathrm{~g}$ anhydrous sodium sulphate, $0.42 \mathrm{~g}$ sodium bicarbonate, $0.36 \mathrm{~g}$ sodium chloride, and $0.18 \mathrm{~g}$ potassium chloride (Isocolan, Bracco, Milan, Italy) in $500 \mathrm{ml}$ of tap water. No antispasmodic or other drugs were given. The protocol included: (1) T2 weighted half Fourier single shot turbo spin echo (HASTE) sequence (TR infinite; TE 90 ms; echo train length 104; section thickness $6 \mathrm{~mm}$; intersection gap 20\%; number of slices 20; effective matrix $192 \times 256$; one signal average) acquired during a single 20 second breath hold; images were acquired every five minutes until the caecum was observed to be filled by the oral contrast agent. (2) Tl weighted fat suppressed spoiled gradient echo sequence (fast low angle shot, FLASH) (TR 160 ms; TE $2.3 \mathrm{~ms}$; section thickness $6 \mathrm{~mm}$; intersection gap 10\%; number of slices 20; effective matrix 140×256; one signal average) acquired during a single breath hold before and 60 seconds following contrast medium administration. Gadolinium chelate (Magnevist; Schering AG, Germany) was administered as a rapid bolus intravenous injection of $0.1 \mathrm{mmol} / \mathrm{kg}$ body weight followed by a normal saline flush. MR images were acquired on both axial and coronal planes. In all patients the average time for complete filling of the ileum ranged from 20 to 25 minutes; furthermore, occupation time of the scanner room was less than 40 minutes for each case.

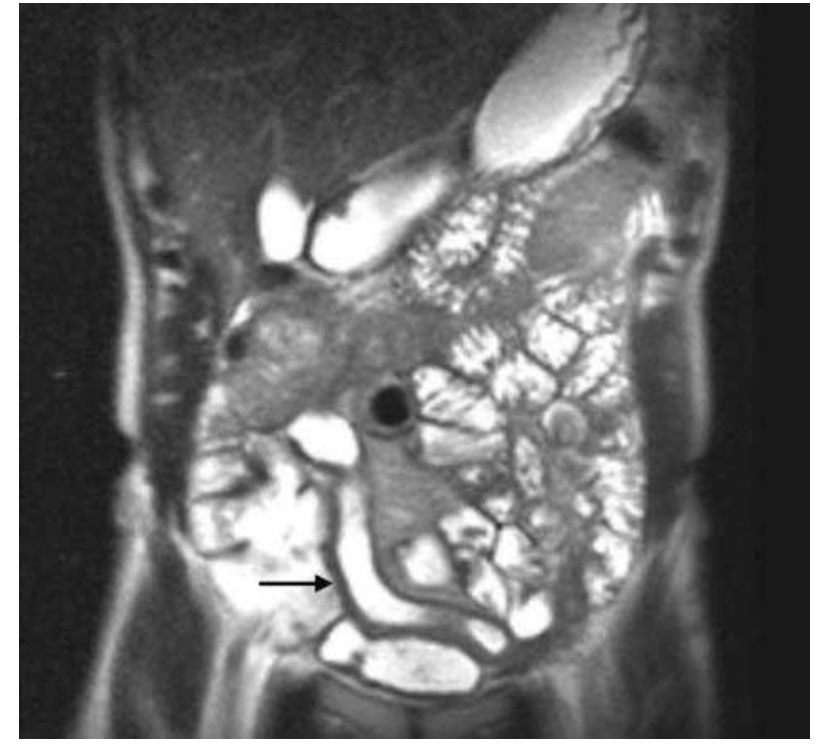

Figure 1 Coronal T2 weighted half Fourier single shot turbo spin echo (HASTE) sequence. The image shows optimal distension of the distal ileum, demonstrating wall thickening and severe irregularities of the internal mucosal surface (arrow).

MRI studies were defined after a consensus review by two experienced radiologists (AL, PP), blinded to endoscopy and histology, both of whom independently completed an MRI data sheet and determined an MRI diagnosis. Image analysis included evaluation of wall thickness and parietal contrast enhancement. A score between 0 and 2 was assigned to each element. Wall thickness $<3 \mathrm{~mm}$ was indicative of a normal bowel and a score of 0 was given; a score of 1 was assigned to a wall thickness of 3.1-6.0 mm and a score of 2 to a wall thickness $>6.0 \mathrm{~mm}$. Contrast enhancement was qualitatively evaluated assigning a score ranging from 0 to $2(0=$ no enhancement; $1=$ mild enhancement; $2=$ marked enhancement). The overall score ranged from 0 (no disease present) to 4 (active disease with severely thickened wall and marked enhancement). Spearman rank correlation was used to compare CE-PEG-MRI, endoscopic, and histological scores as well as CE-PEG-MRI score and CD activity; a p value of less than 0.05 indicated a statistically significant difference. In all cases informed written parental consent for the diagnostic procedures was obtained. The diagnostic approach was approved by the ethics committee of the faculty.

\section{RESULTS}

The final diagnoses were: active CD in 26 cases, active UC in 18 , and indeterminate ileocolitis with spondyloarthropathy (SpA) in $11 ; 20$ children served as controls (eight with irritable bowel syndrome, eight with recurrent abdominal pain, and four with solitary juvenile colonic polyps). Median (range) ages (years) were: $14.0(12-17), 10.0(8-13), 10.6$ (9-13), and $11.2(8-14)$, respectively.

In all $26 \mathrm{CD}$ patients endoscopy and histology revealed inflammation of the distal ileum; in 18 patients several segments of the colon were also affected. In all CD patients CE-PEG- MRI of the distal ileum was markedly abnormal revealing both an increase in wall thickness and parietal contrast enhancement (figs 1, 2A, 2B). In $22 \mathrm{CD}$ patients endoscopic and CE-PEG-MRI scores ranged from 3 to 4 and were coincident in 18; in four patients both endoscopic and CE-PEG-MRI scores were 2. The latter resulted from both an increase in wall thickness and enhanced parietal contrast. CE-PEG-MRI did not reveal involvement of the small bowel proximal to the terminal ileum in any of the CD patients. 

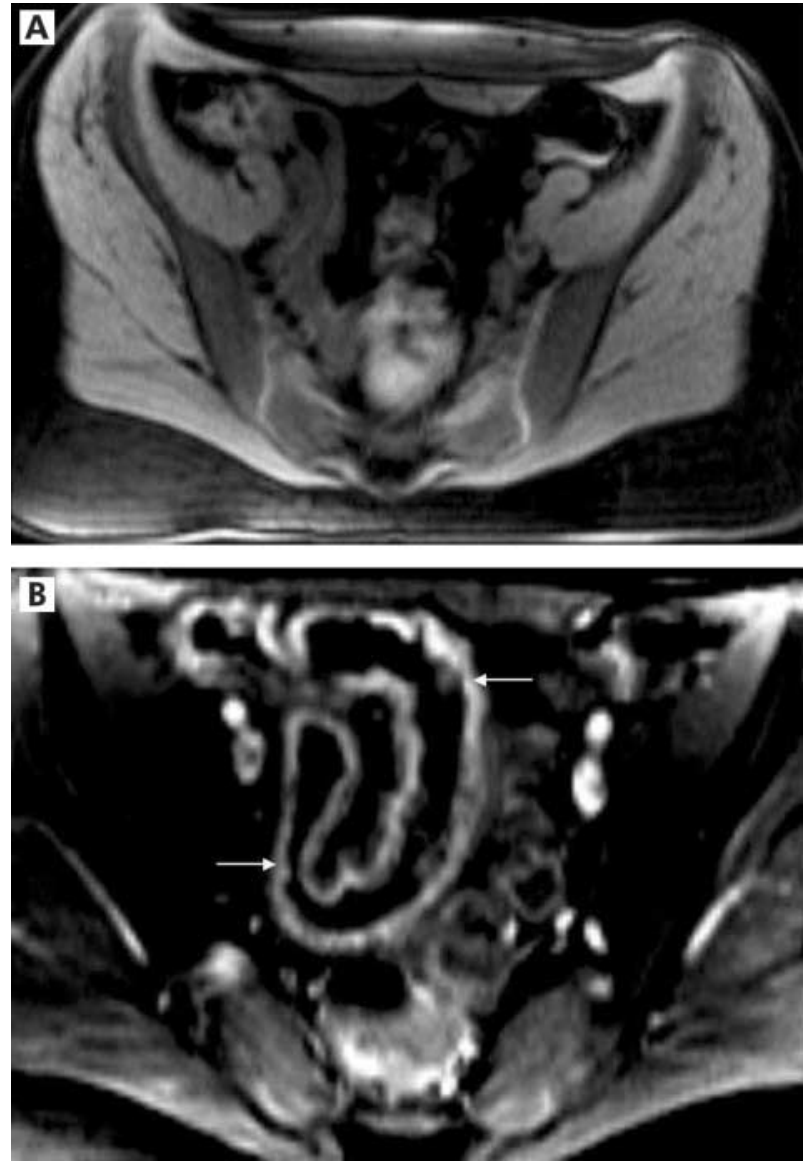

Figure 2 Axial fat suppressed $\mathrm{T} 1$ weighted spoiled gradient echo sequence acquired before $(\mathrm{A})$ and 60 seconds following intravenous administration of gadolinium chelate (B), demonstrating marked parietal enhancement of the distal ileal loop in the contrast enhanced image (arrows).

In seven of the 18 UC patients, endoscopy showed mild ileitis with areas of erythema but no erosions or ulcerations (endoscopic score 1) whereas histology revealed a mild mixed inflammatory infiltrate of the ileal lamina propria without crypt distortion or atrophy, and without epithelial changes. This inflammation ("backwash ileitis") was contiguous to ulcerative inflammation in all colonic segments. In 11 UC patients, the distal ileum was not involved both endoscopically and histologically. CE-PEG-MRI was negative in 15 UC patients and showed a mild parietal contrast enhancement (score 1) without an increase in ileal wall thickness in three of seven patients with backwash ileitis.

Eleven patients received a diagnosis of $\mathrm{SpA}$, with a sacroiliitis documented by radiology and MRI. In six, endoscopy showed ileal hyperaemia, friability, and aphthoid erosions (endoscopic score 2) whereas histology revealed a moderate increase in mononuclear and polymorphonuclear cells in the lamina propria without architectural changes. In five SpA patients, endoscopy showed mild inflammatory changes of the distal ileal mucosa (endoscopic score 1) whereas a mild inflammatory infiltrate in the lamina propria was detected at histology. In all SpA patients several segments of the colon showed endoscopic features such as areas of disappearance of vascular pattern, isolated erosions, and ulcerations, whereas histology detected inflammatory features such as cryptitis, lymphoid aggregates, and increased mixed infiltrate of the lamina propria. CE-PEG-MRI was negative in four SpA patients whereas a mild parietal contrast enhancement without an increase in wall thickness of the distal ileum (score 1)

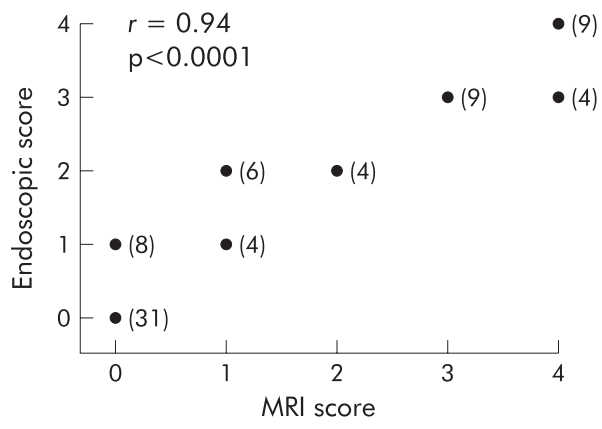

Figure 3 Correlation between endoscopic and contrast enhanced polyethylene glycol magnetic resonance imaging (CE-PEG-MRI) scores in all investigated patients (number of patients in parentheses)

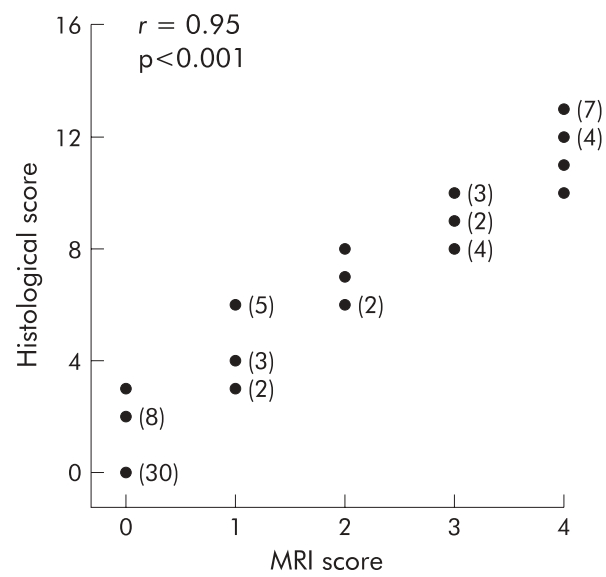

Figure 4 Correlation between histological and contrast enhanced polyethylene glycol magnetic resonance imaging (CE-PEG-MRI) scores in all investigated patients (number of patients in parentheses).

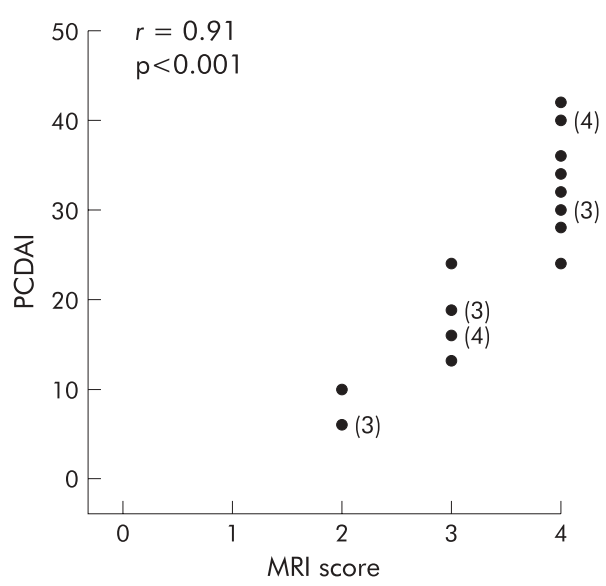

Figure 5 Correlation between paediatric Crohn's disease activity index (PCDAl) and contrast enhanced polyethylene glycol magnetic resonance imaging (CE-PEG-MRI) score in patients with Crohn's disease (number of patients in parentheses).

was observed in the remaining seven patients, six of whom had aphthoid erosions of the distal ileum at endoscopy (score 2).

In all 20 controls both endoscopy and CE-PEG-MRI were negative. In six controls endoscopy showed lymphoid hyperplasia and post-bioptic friability of the distal ileum without erosions or ulcerations. 
In all investigated patients a significant correlation was observed between the CE-PEG-MRI and endoscopic scores $(r=0.94, \mathrm{p}<0.0001)$ (fig 3) as well as between the CE-PEGMRI and histological scores $(r=0.95, \mathrm{p}<0.0001)$ (fig 4$)$. There was also a strong correlation between the CE-PEG-MRI score and PCDAI in CD patients $(r=0.91, \mathrm{p}<0.0001)$ (fig 5). Diagnostic sensitivity and specificity of the CE-PEG-MRI as related to patients with endoscopically documented erosive ileitis were $84 \%$ and $100 \%$, respectively.

\section{DISCUSSION}

Endoscopy is thought to be the most sensitive test for diagnosing IBD by evaluating mucosal changes and extension of intestinal inflammation and by obtaining tissue for histological assessment. As a diagnosis of IBD can often be cumbersome and laborious, the interest of investigators has recently been focused on the development of non-invasive tests to detect ileal inflammation and to differentiate between the various inflammatory diseases of the gut. ${ }^{451213}$

MRI has multiplanar imaging capabilities and can detect inflammatory activity without radiation hazard. ${ }^{14}$ The use of MRI in assessing bowel inflammation has been hindered by technical difficulties such as motion artefacts due to intestinal peristalsis, respiratory motion, and poor resolution. The role of MRI in IBD has also been limited by the relative inexperience of radiologists and clinicians in this area. Recent technical improvements including breath hold acquisition, fat suppression, and the use of oral contrast agents and intravenous gadolinium have made possible high quality MRI of the abdomen. ${ }^{15}$

In our study a solution containing PEG, that is not absorbed or fermented, was used to sufficiently distend the intestinal lumen, a critical variable for intestinal imaging. ${ }^{16}$ In our patients, MRI with the use of an oral contrast agent and bowel wall gadolinium enhancement was a highly sensitive technique in detecting inflammation of the distal ileum. Whereas CE- PEG-MRI was negative in all controls, all children with active CD ileitis had a markedly abnormal CE-PEG-MRI of the terminal ileum. Interestingly, the test revealed both an increase in wall thickness and parietal contrast enhancement in all CD patients, including those with mild to moderate endoscopic lesions (score 2). It is remarkable that strong concordance of both endoscopic and CE-PEG-MRI features was observed in the great majority of patients with active CD. In children with $\mathrm{CD}$, the significantly high correlation between CE-PEG-MRI score and disease activity, as measured by PCDAI, was of outstanding clinical interest. This suggests that the CE-PEG-MRI could be used as a valuable test for monitoring the clinical course of the disease. In addition, it is noteworthy that CE- PEG-MRI also markedly correlated with the histological score of the distal ileal mucosa. Indeed, current expectations from therapy of CD include endoscopic and histological healing of the inflammatory lesions of the mucosa. ${ }^{17}$ Thus our results would also indicate that the test may be useful in monitoring the effect of therapy on intestinal mucosal inflammation.

Our study population included children with inflammatory intestinal diseases other than $\mathrm{CD}$, such as 11 patients with indeterminate ileocolitis associated with SpA. ${ }^{18}$ It is noticeable that CE-PEG-MRI revealed only a mild parietal contrast enhancement of the distal ileum without an increase in wall thickness in six SpA patients with endoscopic aphthoid erosions of the ileal mucosa (score 2) and in one patient with non-specific superficial ileitis (score 1). The latter was also detected at endoscopy in the remaining four patients in whom CE-PEG-MRI was negative.

Determining distribution and extent of IBD is of critical value in differentiating $\mathrm{CD}$ from $\mathrm{UC}$ in order to select appropriate treatment options and direct the follow up programme. ${ }^{19}$ Whereas endoscopic and histological involvement of the ileum is extremely suggestive of $\mathrm{CD}$, endoscopic and histological features of the colon may not be specific enough to always permit differentiation of UC from moderate to severe $\mathrm{CD}^{20}$ Thus exploration of the distal ileum should always be attempted in the diagnostic work up of IBD patients. In our study it was clinically relevant that CE-PEG-MRI did not show a significant increase in ileal wall thickness in any UC patient but revealed mild parietal contrast enhancement in three of seven UC patients with backwash ileitis. The latter is not uncommon in UC patients and consists of patchy microscopic evidence of inflammation without ulcerations, extending a few centimetres into the terminal ileum, in contiguity to colitis of the right colon..$^{21}$ Our data suggest that this condition does not deeply involve the ileal wall as occurs in CD ileitis.

CE-PEG-MRI was negative in six control patients showing lymphoid hyperplasia of the terminal ileum at endoscopy. This picture is often found in children undergoing colonoscopy for chronic unexplained diarrhoea and/or recurrent incapacitating abdominal pain and/or haematochezia. It is commonly viewed as a marker of mucosal reactivity to intestinal bacteria or food antigens and can be radiologically mistaken for an inflammatory infiltrate. ${ }^{22}$ It was noticeable that CE-PEGMRI did not reveal inflammatory changes of the terminal ileum in these patients.

In conclusion, in children with active $\mathrm{CD}$, gadolinium enhanced MRI of the small bowel with the use of an oral PEG solution to distend the small bowel is a highly sensitive and specific test for detecting inflammation of the distal ileum. Our data provide evidence that only CE-PEG-MRI findings, including both increased wall thickness and parietal contrast enhancement of the distal ileum, indicate a transmural ileal inflammation which typically occurs in CD. This test can also be helpful in differentiating active CD from UC and can be proposed in the initial approach to children referred for suspected CD, mainly if presenting symptoms and signs overlap with those of other gastrointestinal disorders. In addition, the test can be of value when the terminal ileum cannot be reached endoscopically because of contraindications and risks. Future studies in CD patients should examine the usefulness of CE-PEG-MRI in monitoring both the clinical course and response to anti-inflammatory therapy.

\section{Authors' affiliations}

A Laghi, P Paolantonio, R Passariello, Department of Radiology, University of Rome "La Sapienza", Rome, Italy

O Borrelli, L Dito, M Bueno de Mesquita, P Falconieri, S Cucchiara, Department of Paediatric Gastroenterology, University of Rome "La Sapienza", Rome, Italy

\section{REFERENCES}

1 Hyams JS. Inflammatory bowel disease. Pediatr Rev 2000;21:291-5.

2 Sawczenko A, Sandhu BK, Logan RF, et al. Prospective survey of childhood inflammatory bowel disease in the British Isles. Lancet 2001;357:1093-4.

3 Griffiths AM, Buller HB. Inflammatory bowel disease. In: Walker WA, Durie PR, Hamilton JR, et al, eds. Pediatric gastrointestinal disease. Pathophysiology, diagnosis, management, 3rd edn. Hamilton: BC Decker Inc, 2000:613-51.

4 Cucchiara S, Celentano L, de Magistris T, et al. Colonoscopy and technetium-99 white cell scan in children with suspected inflammatory bowel disease. J Pediatr 1999;135:727-32.

5 Faure C, Belarbi N, Mougenot JF, et al. Ultrasonographic assessment of inflammatory bowel disease in children: comparison with ileocolonoscopy. J Pediatr 1997:130:147-51.

6 Rieber A, Wruk D, Potthast S, et al. Diagnostic imaging in Crohn's disease: comparison of magnetic resonance imaging and conventional imaging methods. Int J Colorectal Dis 2000;15:176-81.

7 Low RN, Francis IR, Politoske D, et al. Crohn's disease evaluation: comparison of contrast-enhanced MR imaging and single-phase helical CT scanning. J Magn Reson Imaging 2000;11:127-35.

8 Durno CA, Sherman P, Williams T, et al. Magnetic resonance imaging to distinguish the type and severity of pediatric inflammatory bowel diseases. J Pediatr Gastroenterol Nutr 2000;30:170-4. 
9 D'Haens GR, Geboes K, Peeters M, et al. Early lesion of recurrent Cronh's disease caused by infusion of intestinal contents in excluded ileum. Gastroenterology 1998;1 14:262-7.

10 Hyams JS, Ferry GD, Mandel FS, et al. Development and validation of a pediatric Crohn's disease activity index. J Pediatr Gastroenterol Nutr $1991 ; 12: 439-47$

11 Laghi A, Carbone I, Catalano C, et al. Polyethylene glycol solution as an oral contrast agent for MR imaging of the small bowel. Am J Roentgenol 2001;177: 1333-4

12 Weldon MJ, Masoomi AM, Britten AJ, et al. Quantification of inflammatory bowel disease activity using technetium-99m HMPAO labeled leucocyte single photon emission computerized tomography (SPECT). Gut 1995:36:243-50.

13 Parente F, Maconi G, Bollani S, et al. Bowel ultrasound in assessment of Crohn's disease and detection of related small bowel strictures: a prospective comparative study versus $x$ ray and intraoperative findings. Gut 2002;50:490-5

14 Maccioni F, Viscido A, Broglia L, et al. Evaluation of Crohn's disease activity with magnetic resonance imaging. Abdom Imaging 2000;25:219-28.
15 Dassopoulos T. Diagnostic methodologies: serology, endoscopy, and radiology. Curr Gastroenterol Rep 2001:3:491-502.

16 Patak MA, Froehlic JM, von Weymarn C, et al. Non-invasive distension of the small bowel for magnetic-resonance imaging. Lancet 2001;358:978-88.

17 Geboes K, Dalle I. Influence of treatment on morphological features of mucosal inflammation. Gut 2002;50(suppl 3):37-42

18 De Keyser F, Elewaut D, De Vos M, et al. EM. Bowel inflammation and the spondyloarthropathies. Rheum Dis Clin North Am 1998:24:785813.

19 Chutkan RK. Inflammatory bowel disease. Prim Care 2001:28:539-56.

20 Legnani PE, Kornbluth A. Difficult differential diagnoses in IBD: ileitis and indeterminate colitis. Semin Gastrointest Dis 2001;12:21 1-22.

21 Helper DJ, Rex DK. Inflammatory bowel disease. Endoscopy 2001;33:140-6.

22 Wakefield AJ, Murch SH, Anthony A, et al. Ileal-lymphoid-nodular hyperplasia, non- specific colitis, and pervasive developmental disorder in children. Lancet 1998:351:637-41.

23 Yamaue $\mathbf{H}$, Tanimura $\mathrm{H}$, Ishimoto $\mathrm{K}$, et al. Nodular lymphoid hyperplasia of the terminal ileum: report of a case and the findings of an immunological analysis. Surg Today 1996;26:431-4.

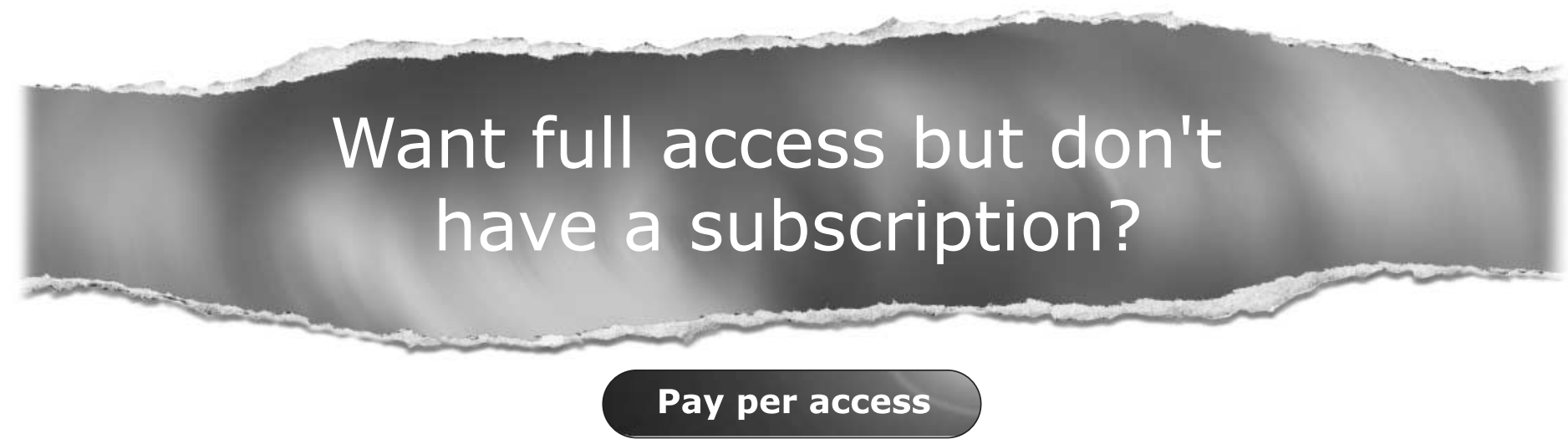

For just US\$25 you can have instant access to the whole website for 30 days. During this time you will be able to access the full text for all issues (including supplements) available. You will also be able to download and print any relevant pdf files for personal use, and take advantage of all the special features Gut online has to offer.

\section{www.gutjnl.com}

